\title{
Strong stimulated Brillouin scattering in an on-chip silicon slot waveguide
}

\author{
Raphaël Van Laer, Dries Van Thourhout and Roel Baets \\ Photonics Research Group, Department of Information Technology, Ghent University - imec \\ $\mathcal{E}$ Center for Nano- and Biophotonics, Ghent University \\ 9000 Ghent, Belgium \\ raphael.vanlaer@intec.ugent.be
}

\begin{abstract}
We analyzed the enhancement of the forward Brillouin gain in a silicon slot waveguide. The calculations predict a gain of $3.6 \times 10^{5} \mathrm{~W}^{-1} \mathrm{~m}^{-1}$, which is an order of magnitude larger than in a silicon wire.
\end{abstract}

(C) 2013 Optical Society of America

OCIS codes: $190.4390,290.5830,120.4880$.

\section{Introduction}

Stimulated Brillouin scattering (SBS) is a nonlinear process which couples optical to mechanical waves [1]. It has a variety of applications, ranging from slow [2] and stored [3] light to Brillouin lasers [4] and frequency comb generation [5,6]. Pant et al. [7] realized on-chip SBS for the first time in chalcogenide waveguides. However, so far it was not possible to observe this effect in silicon wires. The quality factor of the elastic modes was too low because of clamping loss.

Furthermore, it was recently recognized that not just electrostriction but also the gradient force contributes to photon-phonon coupling [8]. Taking both contributions into account, Rakich et al. [8] showed theoretically that an underetched silicon wire can exhibit both forward and backward SBS gains of the order of $10^{4} \mathrm{~W}^{-1} \mathrm{~m}^{-1}$. This is about four orders of magnitude larger than the backward SBS gain in a typical silica fiber. In their work, they described the photon-phonon coupling rigorously by using the optical force distribution $\mathbf{f}$ as input for a mechanical forcedresponse study. In a follow-up [9], they extended that model to allow for the calculation of the SBS gain associated with each mechanical eigenmode separately. The model shows explicitly that the gain near each elastic resonance is proportional to $Q_{m}|\langle\mathbf{f}, \mathbf{u}\rangle|^{2}$, with $Q_{m}$ the mechanical quality factor and $\langle\mathbf{f}, \mathbf{u}\rangle$ the overlap integral between the optical force distribution $\mathbf{f}$ and the mechanical displacement field $\mathbf{u}$. In this paper, we take their model further to the study of silicon slot waveguides - in which large gradient forces are present.

\section{Findings}

Using the model of [9], we investigated the enhancement of the forward SBS gain in a suspended silicon slot waveguide with a very narrow $(5 \mathrm{~nm})$ air-gap, see figures (a) and (b). The horizontal (instead of vertical) slot architecture allows us to go beyond the resolution of e-beam lithography, which limited previous minimum gaps to about $80 \mathrm{~nm}$ [10]. Similar $5 \mathrm{~nm}-$ gap structures have actually been fabricated and are currently used as high-efficiency grating couplers [11].

The small gap leads to an extreme confinement of the fundamental TM-mode, which has an effective index as low as 1.75 at $1.55 \mu \mathrm{m}$. The force density is nearly entirely located on the waveguide boundaries closest to the gap. It has contributions from both electrostriction and the gradient force, but the latter dominates. The total average force on the lower beam is $122 \mathrm{pN} \mathrm{mW}^{-1} \mathrm{\mu m}^{-1}$, which is a factor 8 larger than the highest demonstrated gradient forces [10]. This corresponds to an index sensitivity of $37 \mathrm{RIU} / \mu \mathrm{m}$ and peak-pressures as high as $1395 \mathrm{~Pa} \mathrm{~mW}^{-1}$. Such large optical forces should allow displacement sensitivities below $50 \mathrm{am} \mathrm{Hz}^{-0.5}$.

We considered a situation in which only the lower beam is addressed at its fundamental mechanical eigenfrequency of $3.16 \mathrm{GHz}$. This is a flexural mode with two nodes spaced by $400 \mathrm{~nm}$, 

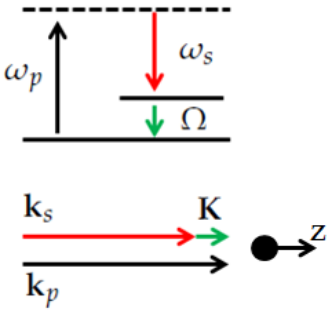

(a) Energy and phase-matching diagrams of forward SBS. The mechanical wavevector $\mathbf{K}$ is very short, so the phonon is practically longitudinally invariant.

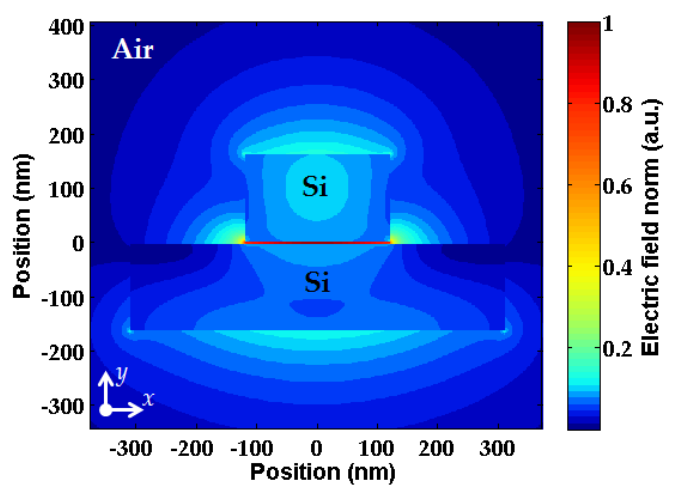

(b) The electric field is confined in the gap between the two silicon beams suspended in air. Both beams are $160 \mathrm{~nm}$ high, the upper one $240 \mathrm{~nm}$ wide and the lower one $620 \mathrm{~nm}$ wide.

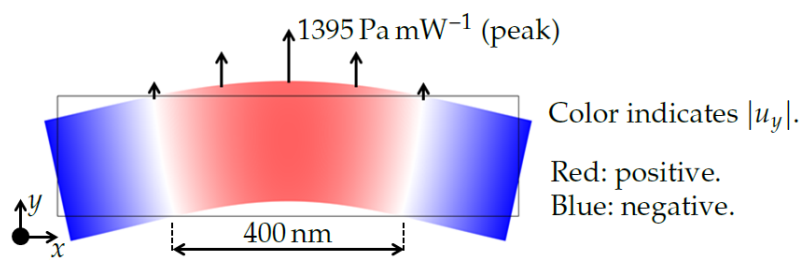

(c) The force distribution located at the upper boundary excites the fundamental elastic mode of the wide beam. The asymmetric configuration (one beam wider) ensures a good photon-phonon overlap.

see figure (c). Since the boundary force density has no nodes, the mechanically excited beam has to be wider to avoid cancellations in the photon-phonon overlap $\langle\mathbf{f}, \mathbf{u}\rangle$. Owing to the strong field confinement in the air-gap, the SBS gain is enhanced to $3.6 \times 10^{5} \mathrm{~W}^{-1} \mathrm{~m}^{-1}-$ a factor 21 larger than the highest value reported for a single silicon wire [9]. Like in [8,9], we assumed a quality factor of 1000. Finally, we have initiated experimental work to verify these predictions.

\section{Conclusion}

Our analysis suggests an order-of-magnitude increase of the forward SBS gain due to the sloteffect in a silicon waveguide. Such high gain may prove vital to the experimental demonstration of low-power (cascaded) SBS on a silicon chip.

Acknowledgement. The research leading to these results has received funding from the European Research Council under the European Union's Seventh Framework Programme - ERC Grant InSpectra.

\section{References}

1. G. P. Agrawal, Nonlinear fiber optics. Academic Press, fifth ed., 2013.

2. Y. Okawachi et al. Physical Review Letters, vol. 153902, pp. 1-4, 2005.

3. Z. Zhu et al. Science, vol. 318, pp. 1748-50, Dec. 2007.

4. L. F. Stokes et al. Optics Letters, vol. 7, pp. 509-11, Oct. 1982.

5. D. Braje et al. Physical Review Letters, vol. 102, pp. 1-4, May 2009.

6. M. S. Kang et al. Nature Physics, vol. 5, pp. 276-280, Mar. 2009.

7. R. Pant et al. Optical Express, pp. 145-146, 2011.

8. P. Rakich et al. Physical Review X, vol. 2, pp. 1-15, Jan. 2012.

9. W. Qiu et al. arXiv:1210.0267, vol. 5865, no. 2006, pp. 2764-2777, 2012.

10. M. Li et al. Applied Physics Letters, vol. 97, no. 18, p. 183110, 2010.

11. G. Roelkens et al. Optics Express, vol. 14, pp. 11622-30, Nov. 2006. 